\title{
Presentations of semigroup algebras of weighted trees
}

\author{
Christopher Manon
}

Received: 18 September 2008 / Accepted: 19 June 2009 / Published online: 16 July 2009

(c) The Author(s) 2009. This article is published with open access at Springerlink.com

\begin{abstract}
We study presentations for subalgebras of invariants of the coordinate algebras of binary symmetric models of phylogenetic trees studied by Buczynska and Wisniewski in (J. Eur. Math. Soc. 9:609-635, 2007). These algebras arise as toric degenerations of projective coordinate rings of the moduli of weighted points on the projective line, and projective coordinate rings of the moduli of quasiparabolic semisimple rank two bundles on the projective line.
\end{abstract}

Keywords Toric ideal $\cdot$ Polytope $\cdot$ Phylogenetics

\section{Introduction}

Let $\mathcal{T}$ be an abstract trivalent tree with leaves $V(\mathcal{T})$, edges $E(\mathcal{T})$, and non-leaf vertices $I(\mathcal{T})$, by trivalent we mean that the valence of $v \in I(\mathcal{T})$ is always three. Let $e_{i}$ be the unique edge incident to the leaf $i \in V(\mathcal{T})$. Let $Y$ be the unique trivalent tree with three leaves. For each $v \in I(\mathcal{T})$ we pick an injective map $i_{v}: Y \rightarrow \mathcal{T}$, sending the unique member of $I(Y)$ to $v$. We denote the members of $E(Y)$ by $E, F$, and $G$. We say that two leaves $e, f \in V(\mathcal{T})$ are paired if they are connected to a common internal vertex. Members of $V(\mathcal{T})$ which are not paired are called lone leaves. We will be concerned with properties of weightings of trivalent trees, defined as a functions

$$
\omega: E(\mathcal{T}) \rightarrow \mathbb{Z}_{\geq 0}
$$

The author was supported by the NSF FRG grant DMS-0554254. The author will include these results in his doctoral thesis.

C. Manon $(\bowtie)$

Department of Mathematics, University of Maryland, College Park, MD 20742, USA

e-mail: manonc@math.umd.edu 
The maps $i_{v}$ define pull-back operations on weightings by the formulas

$$
\begin{aligned}
& i_{v}^{*}(\omega)(E)=\omega\left(i_{v}(E)\right), \\
& i_{v}^{*}(\omega)(F)=\omega\left(i_{v}(F)\right), \\
& i_{v}^{*}(\omega)(G)=\omega\left(i_{v}(G)\right) .
\end{aligned}
$$

Definition 1.1 Let $S_{\mathcal{T}}$ be the graded semigroup where $S_{\mathcal{T}}[k]$ is the set of weightings which satisfy the following conditions.

(1) For all $v \in I(\mathcal{T})$ the numbers $i_{v}^{*}(\omega)(E), i_{v}^{*}(\omega)(F)$ and $i_{v}^{*}(\omega)(G)$ satisfy

$$
\left|i_{v}^{*}(\omega)(E)-i_{v}^{*}(\omega)(F)\right| \leq i_{v}^{*}(\omega)(G) \leq\left|i_{v}^{*}(\omega)(E)+i_{v}^{*}(\omega)(F)\right| .
$$

These are referred to as the triangle inequalities.

(2) $i_{v}^{*}(\omega)(E)+i_{v}^{*}(\omega)(F)+i_{v}^{*}(\omega)(G)$ is even.

(3) $\sum_{i \in V(\mathcal{T})} \omega\left(e_{i}\right)=2 k$.

Note that because the triangle inequalities hold for the integers $i_{v}^{*}(\omega)(E)$, $i_{v}^{*}(\omega)(F)$, and $i_{v}^{*}(\omega)(G)$ if and only if a triangle exists with these side lengths, the condition is symmetric in $E, F$, and $G$. In [8] Speyer and Sturmfels show that the semigroup algebras $\mathbb{C}\left[S_{\mathcal{T}}\right]$ may be realized as projective coordinate rings of flat toric deformations of $G r_{2}\left(\mathbb{C}^{n}\right)$ where $n=|V(\mathcal{T})|$, for the Plücker embedding. This semigroup is also multigraded, with the grading given by the weights $\omega\left(e_{i}\right)$ on the leaf edges of the tree. For a vector of non-negative integers $\mathbf{r}=\left(r_{1}, \ldots, r_{n}\right)$ we let $S_{\mathcal{T}}[\mathbf{r}]$ be the set of weightings $\omega \in S_{\mathcal{T}}$ with $\omega\left(e_{i}\right)=r_{i}$.

Definition 1.2 Let $\mathbf{r}: V(\mathcal{T}) \rightarrow \mathbb{Z}_{\geq 0}$ be a vector of non-negative integers. Let $S_{\mathcal{T}}(\mathbf{r})$ be the multigraded subsemigroup of $S_{\mathcal{T}}$ formed by the pieces $S_{\mathcal{T}}[k \mathbf{r}]$.

In general we will focus on weightings of trivalent trees such that the vector of edge weights $\mathbf{r}$ has an even total sum, because of the following proposition. The proof provides a nice introduction to the study of weights on trivalent trees.

Proposition 1.3 Let $\mathcal{T}$ be a trivalent tree. If $\mathbf{r}$ has an odd total sum, then there is no weighting of $\mathcal{T}$ satisfying the parity condition with edges weighted by $\mathbf{r}$.

Proof Note that this is true by definition for $\mathcal{T}=Y$. Suppose that the result holds for every trivalent tree with $n-1$ leaves, and consider a $\mathcal{T}$ with $n$ leaves. Pick paired leaves $e, f$ in $V(\mathcal{T})$, and let $\mathcal{T}^{\prime}$ be the trivalent tree obtained by forgetting $e$ and $f$, and the edges connected to them. Let $g$ be the internal edge of $\mathcal{T}$ which shares a vertex with $f$ and $g$. Note that we may consider $g$ a leaf of $\mathcal{T}^{\prime}$. Any weighting $\omega$ which satisfies parity also defines a weighting of $\mathcal{T}^{\prime}$ by restriction. By the induction hypothesis, $\omega \mid \mathcal{T}^{\prime}$ weights an even number of $V\left(\mathcal{T}^{\prime}\right)$ with odd numbers. There are two cases, if $g$ is weighted odd then by parity only one of $e$ or $f$ may be weighted odd. If $g$ is weighted even, then either both $e$ and $f$ are weighted odd, or neither is weighted 
odd. This shows that any weighting of $\mathcal{T}$ must have leaf weights which sum to an even number.

It follows from work in [8] that graded algebras $\mathbb{C}\left[S_{\mathcal{T}}(\mathbf{r})\right]$ are homogeneous coordinate rings for projective embeddings of flat toric deformations of $G r_{2}\left(\mathbb{C}^{n}\right) / / \mathbf{r} T$, the weight variety of the Grassmannian of 2-planes associated to $\mathbf{r}$, or equivalently $\mathcal{M}_{\mathbf{r}}$, the moduli space of $\mathbf{r}$-weighted points on $\mathbb{C} P^{1}$ (see [6] for this connection). In [6] this degeneration is used to construct presentations of the projective coordinate ring of $\mathcal{M}_{\mathbf{r}}$ for the Plücker embedding, and it is shown that these algebras are generated in degree 1 and have relations generated by quadrics and cubics for certain $\mathcal{T}$ and $\mathbf{r}$. This is the starting point for the present paper. Forgetting the grading for a moment, geometrically $S_{\mathcal{T}}$ is the semigroup of lattice points in a cone $P_{\mathcal{T}}$ in $\mathbb{R}^{|E(\mathcal{T})|}$. The inequalities defining $P_{\mathcal{T}}$ are given by the triangle inequalities, and the parity condition defines a certain sublattice of $\mathbb{Z}^{|E(\mathcal{T})|}$. Let $\mathcal{T}_{1}$ and $\mathcal{T}_{2}$ be trivalent trees with $N_{1}$ and $N_{2}$ leaves, respectively. Identify the leaf 1 from $\mathcal{T}_{2}$ with the leaf $N_{1}$ from $\mathcal{T}_{1}$, relabelling the leaves of $\mathcal{T}_{2}$ as follows, $2 \rightarrow N_{1}+1, \ldots, N_{2} \rightarrow N_{1}+N_{2}-1$. This creates a tree with a unique vertex of valence 2 , replace this vertex and both of its incident edges with a single edge, the resulting tree $\mathcal{T}_{1} * \mathcal{T}_{2}$ is trivalent. We call this operation merging, see Figure 1 for an example. Let $i \in V(\mathcal{T})$, and denote the projection onto the $e_{i}$-th component of $\mathbb{R}^{|E(\mathcal{T})|}$ by $\pi_{i}$. It is simple to check that

$$
P_{\mathcal{T}_{1} * \mathcal{T}_{2}}=P_{\mathcal{T}_{1}} \times_{\pi_{N_{1}}=\pi_{1}} P_{\mathcal{T}_{2}} .
$$

Where the right hand side is the fibered product of the polytopes $P_{\mathcal{T}_{1}}$ and $P_{\mathcal{T}_{2}}$ over the maps $\pi_{N_{1}}$ and $\pi_{1}$. In particular this implies that all $P_{\mathcal{T}}$ are fibered products of copies of $P_{Y}$. This is reminiscent of the theory of moduli of structures on orientable surfaces, where structures on a surface of high genus can be glued together from structures on three-punctured spheres over a pair-of-pants decomposition. The reason for this resemblance is not entirely accidental, see [5] for a moduli-of-surfaces interpretation of spaces associated to the semigroup $S_{\mathcal{T}}$. Buczynska and Wisniewski define merging in [3], where they show that a similar fibered product formula holds for a class of semigroups of weightings which we will now introduce.

Definition 1.4 For a trivalent tree $\mathcal{T}$ let $\Delta(\mathcal{T})$ be the polytope in $\mathbb{R}^{|E(\mathcal{T})|}$ formed by the convex hull of weightings $\omega$ such that $\omega(e) \in\{0,1\}$ for all $e \in E(\mathcal{T})$, and $i_{v}^{*}(\omega)(E)+i_{v}^{*}(\omega)(F)+i_{v}^{*}(\omega)(G) \in 2 \mathbb{Z}$ for all $v \in I(\mathcal{T})$.

It is shown in [3] (proposition 1.13) that $\Delta(\mathcal{T})$ is a fibered product of $|I(\mathcal{T})|$ copies of $\Delta(Y)$. The lattice point semigroup of $L \Delta(\mathcal{T})=\Delta(\mathcal{T})+\ldots+\Delta(\mathcal{T})$ is isomorphic to the following semigroup.

Definition 1.5 Let $L$ be a positive integer. Let $S_{\mathcal{T}}^{L}$ be the graded semigroup where $S_{\mathcal{T}}^{L}[k]$ is the set of weightings $\omega$ of $\mathcal{T}$ which satisfy

(1) For all $v \in I(\mathcal{T})$ the numbers $i_{v}^{*}(\omega)(E), i_{v}^{*}(\omega)(F)$ and $i_{v}^{*}(\omega)(G)$ satisfy the triangle inequalities.

(2) $i_{v}^{*}(\omega)(E)+i_{v}^{*}(\omega)(F)+i_{v}^{*}(\omega)(G)$ is even. 
(3) $i_{v}^{*}(\omega)(E)+i_{v}^{*}(\omega)(F)+i_{v}^{*}(\omega)(G) \leq 2 k L$.

This last condition is referred to as the level condition.

Note that $S_{\mathcal{T}}^{1}$ has a fibered product decomposition into copies $S_{Y}^{1}$ in a way completely analogous to $S_{\mathcal{T}}$. To see that the lattice points of $\Delta(\mathcal{T})$ correspond with the first graded piece of $S_{\mathcal{T}}^{1}$, one need only use the fibered product decomposition of both objects. We observe that the lattice points of $\Delta(Y)$ are given by the degree 1 members of $S_{Y}^{1}$. In [3] Buczynska and Wisniewski study the algebras $\mathbb{C}\left[S_{\mathcal{T}}^{1}\right]$, proving that they are all deformation equivalent. However, they do not construct an analogue of the projective coordinate ring of the Grassmannian of two planes in this context, namely an algebra for each $n$ which flatly degenerates to the semigroup algebra $\mathbb{C}\left[S_{\mathcal{T}}^{1}\right]$ for each tree $\mathcal{T}$ with $n$ leaves while preserving the multigrading defined by the edge weights and the level.

In [10], Sturmfels and Xu show how to flatly deform the multigraded Cox-Nagata ring $R^{G}$ to each $\mathbb{C}\left[S_{\mathcal{T}}^{1}\right]$. The structure of this ring was studied by Castravet and Tevelev in [4] and by Mukai in [7]. Let $R$ be a polynomial ring over $\mathbb{C}$ in $2 n$ variables,

Fig. 1 Merging two trees
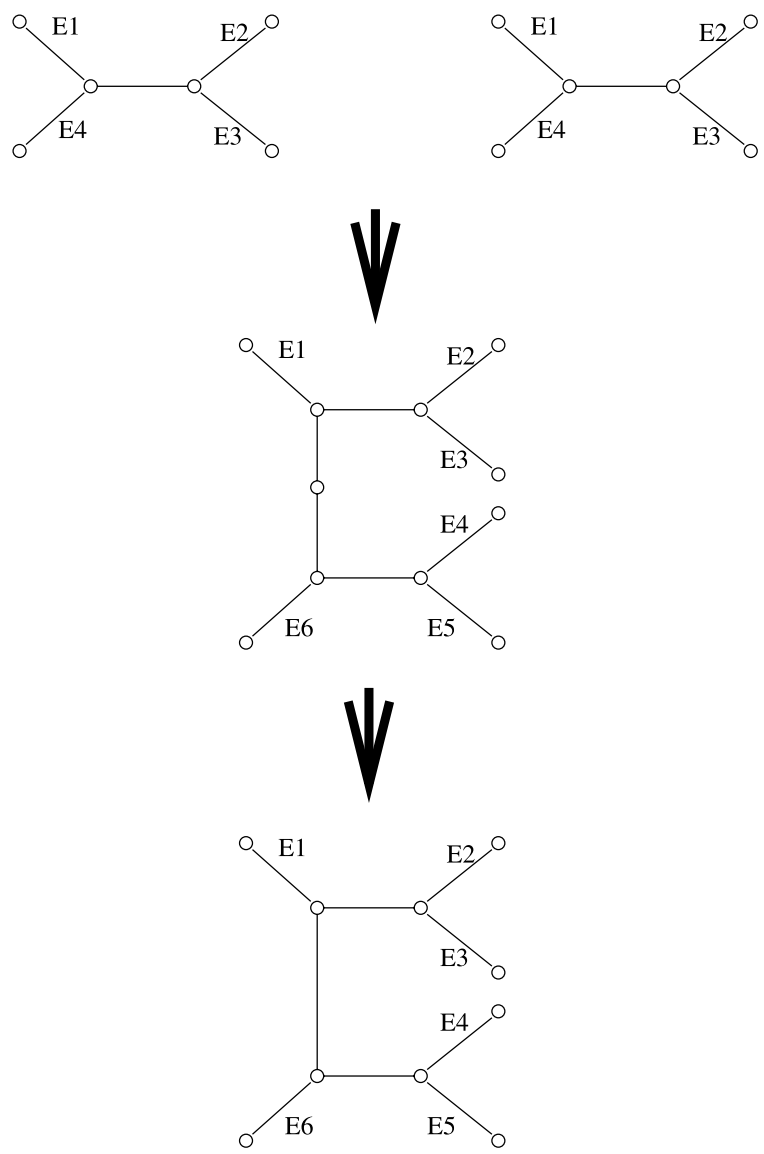
and let $G$ be a subspace of $\mathbb{C}^{n}$ of codimension $d$. There is an action of $G$ on $R$ which generalizes the construction used by Nagata in his solution to Hilbert's 14th problem. When $d \geq 3$ the invariant ring $R^{G}$ is isomorphic to the Cox ring of $X_{G}$, the blow-up of $\mathbb{P}^{d-1}$ at $n$ points determined by $G$, see [10] for a discussion of this connection. Let $K$ be the pullback of the canonical class on $\mathbb{P}^{d-1}$, and let the $E_{i}$ be the associated distinguished classes of the blow-up. Then

$$
\operatorname{Cox}\left(X_{G}\right)=\bigoplus_{(\vec{u}, r)} H^{0}\left(X_{G}, r K+\left(u_{1}-r\right) E_{1}+\ldots+\left(u_{n}-r\right) E_{n}\right) .
$$

Sturmfels and $\mathrm{Xu}$ prove the following theorem.

Theorem 1.6 If $G$ is generic and of codimension $2, R^{G}$ flatly degenerates to $\mathbb{C}\left[S_{\mathcal{T}}^{1}\right]$ for each tree $\mathcal{T}$ with $n$ leaves.

The ring $R^{G}$ comes equipped with a multigrading given by $(r, \vec{u}) \in \mathbb{Z}^{n+1}$. We relabel the $(r, \vec{u})$ component of $R^{G}$ with the multigrade $(\mathbf{r}, L)$ with $L=\left(\sum_{i=1}^{n} u_{i}\right)-r$, $r_{1}=u_{1}, \ldots, r_{n-1}=u_{n-1}$, and $r_{n}=\left(\sum_{i=1}^{n} u_{i}\right)-r-u_{n}$. This multigrade agrees with the one on $\mathbb{C}\left[S_{\mathcal{T}}^{1}\right]$ induced by the level $L$ and the leaf weights under the flat deformation, see [10] for details. Since the multigrading is given by the Picard Group of the blow-up, we refer to it as the Picard grading, and we refer to the associated torus acting on the ring as the Picard torus. The analogue of the projective coordinate rings $\mathbb{C}\left[\mathcal{M}_{\mathbf{r}}\right]$ in this context are the multigrade $(\mathbf{r}, L)$ Veronese subrings $R^{G}(\mathbf{r}, L)$, obtained by taking the direct sum of all components of $R^{G}$ with multigrade a multiple of $(\mathbf{r}, L)$. These are the projective coordinate rings of the embeddings of $X_{(n-3, n)}$, the blow-up of $\mathbb{P}^{n-3}$ at $n$ points, corresponding to various members of $\operatorname{Pic}\left(X_{(n-3, n)}\right)$. Let $S_{\mathcal{T}}^{L}(\mathbf{r})$ be the subsemigroup of $S_{\mathcal{T}}^{1}$ of pieces with multigrade a multiple of $(\mathbf{r}, L)$. It follows that $\mathbb{C}\left[S_{\mathcal{T}}^{L}(\mathbf{r})\right]$ is a toric deformation of $R^{G}(\mathbf{r}, L)$. Sturmfels and Xu point out that the ring $\mathbb{C}\left[G r_{2}\left(\mathbb{C}^{n}\right)\right]$ is isomorphic as a multigraded algebra to $\operatorname{Cox}\left(X_{(n-3, n-1)}\right)$. This gives a common interpretation of both types of semigroups of weighted trees considered here as toric deformations of projective coordinate rings associated to embeddings of blow-ups of projective spaces. It was also noted in [10] that by a theorem of Bauer [2], $X_{(n-3, n)}$ is related to $N_{(0, n)}$, the moduli space of quasiparabolic semistable rank 2 bundles on $\mathbb{P}^{1}$, by a sequence of flops. This implies that these spaces share the same Cox ring, and that the algebras $\mathbb{C}\left[S_{\mathcal{T}}^{L}(\mathbf{r})\right]$ are toric deformations of the projective coordinate rings associated to line bundles on $N_{(0, n)}$.

We study the degrees of presentation and relation generation for presentations of a large class of the rings $\mathbb{C}\left[S_{\mathcal{T}}^{L}(\mathbf{r})\right]$, finding upper bounds for both of these numbers. The techniques used are such that the same results immediately hold for $\mathbb{C}\left[S_{\mathcal{T}}(\mathbf{r})\right]$ as well, in particular we give a different proof of a fundamental result of [6] on a presentation of these rings. It follows that the same bounds on generator and relation degrees also apply to $R^{G}(\mathbf{r}, L)$ when $G$ is of codimension 2 and 1 , and $\mathbb{C}\left[\mathcal{M}_{\mathbf{r}}\right]$ as well. In particular our results apply to the projective coordinate rings of a large class of embeddings of blow-ups of $\mathbb{P}^{n-3}$ at $n$ and $n-1$ points. From now on we assume $G$ is of codimension 2 unless otherwise indicated. 


\subsection{Statement of Results}

We now state the main results of the paper. We will be focusing on the following class of $S_{\mathcal{T}}^{L}(\mathbf{r})$.

Definition 1.7 We call the triple $(\mathcal{T}, \mathbf{r}, L)$ admissible if $L$ is even, $\mathbf{r}(i)$ is even for every lone leaf $i$, and $\mathbf{r}(j)+\mathbf{r}(k)$ is even for all paired leaves $j, k$.

Remark 1.8 The assumption that $\mathbf{r}$ has an even total sum implies that an even number, $2 M$ of the entries of $\mathbf{r}$ are odd. Choosing $\mathcal{T}$ with $2 M$ paired leaves then guarantees that $(\mathcal{T}, \mathbf{r}, L)$ is admissible, provided that $L$ is even. This is important for constructing presentations of $R^{G}(\mathbf{r}, L)$, since this ring always has a flat deformation to $\mathbb{C}\left[S_{\mathcal{T}}^{L}(\mathbf{r})\right]$ for some admissible $(\mathcal{T}, \mathbf{r}, L)$ when $L$ is even. Also note that the second Veronese subring of $\mathbb{C}\left[S_{\mathcal{T}}^{L}(\mathbf{r})\right]$ is the semigroup algebra associated to $(\mathcal{T}, 2 \mathbf{r}, 2 L)$, which is always admissible.

Theorem 1.9 For $(\mathcal{T}, \mathbf{r}, L)$ admissible with $L>2, \mathbb{C}\left[S_{\mathcal{T}}^{L}(\mathbf{r})\right]$ is generated in degree 1 .

Theorem 1.10 For $(\mathcal{T}, \mathbf{r}, L)$ admissible with $L>2, \mathbb{C}\left[S_{\mathcal{T}}^{L}(\mathbf{r})\right]$ has relations generated in degree at most 3.

As a corollary we get the same results for $S_{\mathcal{T}}(\mathbf{r})$ when $(\mathcal{T}, \mathbf{r})$ satisfy admissibility conditions. These theorems are proved in Sections 2, 3, and 4. In Section 5 we look at some special cases, and investigate what can go wrong when $(\mathcal{T}, \mathbf{r}, L)$ is not an admissible triple.

\subsection{Outline of techniques}

To prove Theorems 1.9 and 1.10 we use two main ideas. First, we employ the following trivial but useful observation.

Proposition 1.11 Let $(\mathcal{T}, \mathbf{r}, L)$ be admissible, then for any weighting $\omega \in S_{\mathcal{T}}^{L}(\mathbf{r})$, $\omega(e)$ is an even number when $e$ is not an edge connected to a paired leaf.

This allows us to drop the parity condition that $i_{v}^{*}(\omega)(E)+i_{v}^{*}(\omega)(F)+i_{v}^{*}(\omega)(G)$ is even by forgetting the paired leaves and halving all remaining weights.

Fig. 2 Clipping the paired leaves of $\mathcal{T}$

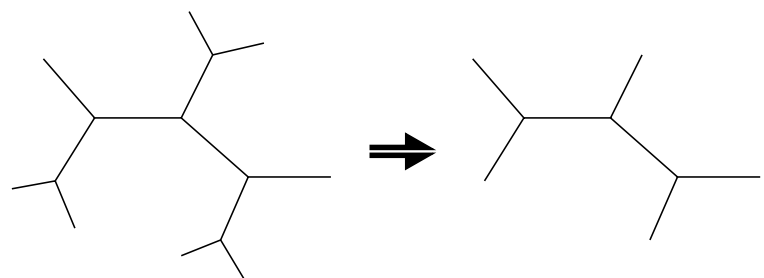


Definition 1.12 Let $c(\mathcal{T})$ be the subtree of $\mathcal{T}$ given by forgetting all edges incident to paired leaves. We call $c(\mathcal{T})$ a clipping of $\mathcal{T}$.

Definition 1.13 Let $U_{c(\mathcal{T})}^{L}(\mathbf{r})$ be the graded semigroup of weightings on $c(\mathcal{T})$ such that the members of $U_{c(\mathcal{T})}^{L}(\mathbf{r})[k]$ satisfy the triangle inequalities, the new level condition $i_{v}^{*}(\omega)(E)+i_{v}^{*}(\omega)(F)+i_{v}^{*}(\omega)(G) \leq k L$, and the following conditions.

1. $\omega\left(e_{i}\right)=k \frac{\mathbf{r}(i)}{2}$ for $i$ a lone leaf of $\mathcal{T}$.

2. $\frac{k|\mathbf{r}(i)-\mathbf{r}(j)|}{2} \leq \omega(e) \leq \frac{k|\mathbf{r}(i)+\mathbf{r}(j)|}{2}$ for e the unique edge of $\mathcal{T}$ connected to the vertex which is connected to the paired leaves $i$ and $j$.

3. $\omega(e)+\frac{k \mathbf{r}(i)+k \mathbf{r}(j)}{2} \leq k L$.

Also, let $U_{c(\mathcal{T})}^{L}$ be the graded semigroup of weightings which satisfy the triangle inequalities and the new level condition for $L$. The following is a consequence of these definitions.

Proposition 1.14 For $(\mathcal{T}, \mathbf{r}, L)$ admissible,

$$
U_{c(\mathcal{T})}^{L}(\mathbf{r}) \cong S_{\mathcal{T}}^{L}(\mathbf{r})
$$

as graded semigroups.

The next main idea is to undertake the analysis of $U_{c(\mathcal{T})}^{L}(\mathbf{r})$ by first considering the weightings $i_{v}^{*}(\omega) \in U_{Y}^{L}$. After constructing an object in $U_{Y}^{L}$, like a factorization or a relation, we "glue" these objects back together along edges shared by the various $i_{v}(Y)$ with the fibered product. We obtain information about $U_{Y}^{L}$ by studying the geometry of the following polytope. Let $P_{3}(L)$ be the convex hull of $(0,0,0)$, $\left(\frac{L}{2}, \frac{L}{2}, 0\right),\left(\frac{L}{2}, 0, \frac{L}{2}\right)$, and $\left(0, \frac{L}{2}, \frac{L}{2}\right)$.

The graded semigroup of lattice points for $P_{3}(L)$ is clearly $U_{Y}^{L}$. By a lattice equivalence of polytopes $P, Q \subset \mathbb{R}^{n}$ with respect to a lattice $\Lambda \subset \mathbb{R}^{n}$ we mean a composition of translations by members of $\Lambda$ and members of $G L(\Lambda) \subset G L_{n}(\mathbb{R})$ which takes $P$ to $Q$. If $P$ and $Q$ are lattice equivalent it is easy to show that they have isomorphic graded semigroups of lattice points. When $L$ is an even integer (admissibility condition) the intersection of this polytope with any translate of the unit cube in $\mathbb{R}^{3}$, is, up to lattice equivalence, one of the polytopes shown in Figure 4.

In Section 2 we show that these polytopes are normal, and the relations of their associated semigroups are generated in degree at most 3. In Sections 3 and 4 we will lift these properties to $U_{c(\mathcal{T})}^{L}(\mathbf{r})$, and therefore $S_{\mathcal{T}}^{L}(\mathbf{r})$ for $(\mathcal{T}, \mathbf{r}, L)$ admissible. Facts about the six polytopes above also allow us to carry out a more detailed study of the semigroups $S_{\mathcal{T}}^{L}(\mathbf{r})$ in Section 5, for example they allow us to show the redundancy of the cubic relations for certain $(\mathcal{T}, \mathbf{r}, L)$.

We thank John Millson for introducing us to this problem, Ben Howard for many useful and encouraging conversations and for first introducing us to the commutative algebra of semigroup rings, Larry O'Neil for several useful conversations on the cone of triples which satisfy the triangle inequalities, the referees for many useful suggestions, and Bernd Sturmfels for introducing us to Graver bases and shortening the proof of Theorems 2.2 and 2.4. 
Fig. $3 P_{3}(2 L)$

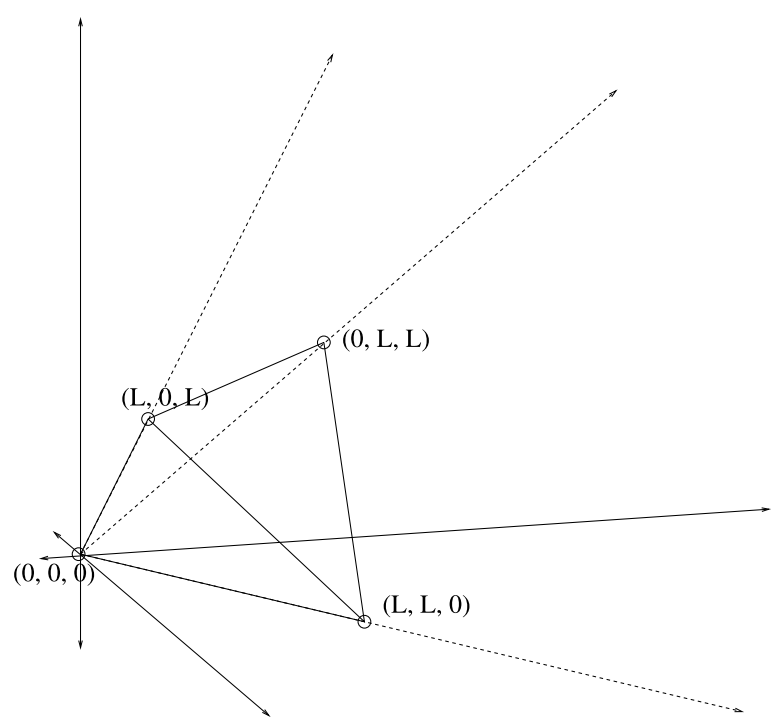

Fig. 4 Cube Polytopes
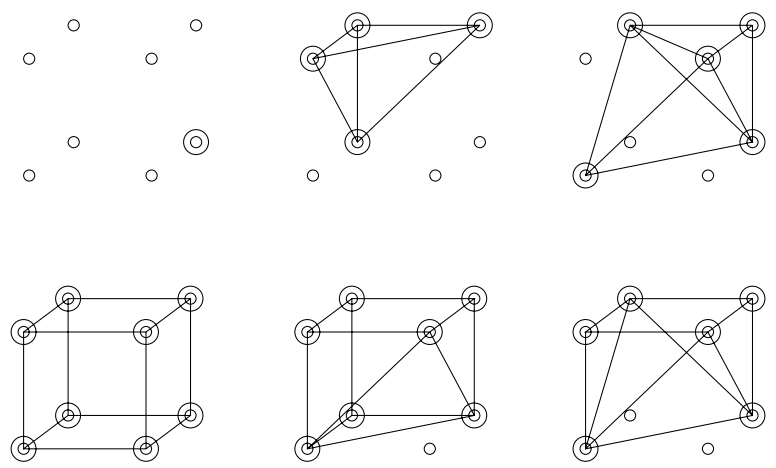

\section{The Cube Semigroups}

In this section we prove that the intersection of any translate of the unit cube of $\mathbb{R}^{3}$ with $P_{3}(L)$ produces a normal polytope whose semigroup of lattice points has relations generated in degree at most 3 when $L$ is even. Let $P_{3}$ be the cone of triples of nonnegative integers which satisfy the triangle inequalities, and let $C\left(m_{1}, m_{2}, m_{3}\right)$ denote the unit cube rooted at $\left(m_{1}, m_{2}, m_{3}\right) \in \mathbb{R}^{3}$,

$$
C\left(m_{1}, m_{2}, m_{3}\right)=\operatorname{conv}\left\{\left(m_{1}+\epsilon_{1}, m_{2}+\epsilon_{2}, m_{3}+\epsilon_{3}\right) \mid \epsilon_{i} \in\{0,1\}\right\} .
$$

We wish to classify the polytopes which have the presentation $C\left(m_{1}, m_{2}, m_{3}\right) \cap P_{3}$. Since $P_{3}$ is symmetric we may assume that $\left(m_{1}, m_{2}, m_{3}\right)$ is ordered by magnitude with $m_{3}$ the largest. In this analysis we keep track of the triangle inequalities with the quantities $n_{i}=m_{j}+m_{k}-m_{i}$. For example, a point $\left(m_{1}, m_{2}, m_{3}\right)$ is a member of $P_{3}$ 
if $n_{i} \geq 0$ for each $i$. Immediately we have the following inequalities.

$$
n_{1} \geq n_{2} \geq n_{3}, n_{2} \geq 0
$$

If $n_{3}<-2$ then no member of $C\left(m_{1}, m_{2}, m_{3}\right)$ can belong to $P_{3}$. If $n_{3} \geq-2$ then there are six distinct possibilities, we list each case along with the standard lattice members of $C\left(m_{1}, m_{2}, m_{3}\right) \cap P_{3}-\left(m_{1}, m_{2}, m_{3}\right)$.

\begin{tabular}{|r|r|}
\hline Condition & $C\left(m_{1}, m_{2}, m_{3}\right) \cap P_{3}-\left(m_{1}, m_{2}, m_{3}\right)$ \\
\hline$n_{3}=-2$ & $(1,1,0)$ \\
$n_{3}=-1$ & $(1,1,0),(0,1,0),(1,0,0),(1,1,1)$ \\
$n_{1}=n_{2}=n_{3}=0$ & $(1,1,0),(0,1,1),(1,0,1),(1,1,1),(0,0,0)$ \\
$n_{1}>0, n_{2}=n_{3}=0$ & $(1,1,0),(0,1,1),(1,0,1),(1,1,1),(0,0,0),(0,0,1)$ \\
$n_{1}, n_{2}>0, n_{3}=0$ & $(1,1,0),(0,1,1),(1,0,1),(1,1,1),(0,0,0),(0,0,1),(0,1,0)$ \\
$n_{i}>0$ & all lattice points of the cube \\
\hline
\end{tabular}

The figure below illustrates these arrangements.

Now we will see what happens when we intersect $P_{3}$ with the half space defined by the inequality $v_{1}+v_{2}+v_{3} \leq 2 L$ to get $P_{3}(2 L)$. The reader may want to refer to Figure 6 for this part. The convex set $C\left(m_{1}, m_{2}, m_{3}\right) \cap P_{3}(2 L)$ can be one of the above polytopes (up to lattice equivalence), or one of them intersected with the half plane $v_{1}+v_{2}+v_{3} \leq 2 L$. Note that a vertex $v$ in $C\left(m_{1}, m_{2}, m_{3}\right) \cap P_{3}(2 L)$ lying on a facet of $P_{3}$ necessarily satisfies $v_{1}+v_{2}+v_{3}=0 \bmod 2$. In Figure 6 these points are colored black.

The hyperplane defined by the equation $v_{1}+v_{2}+v_{3}=2 L$ must intersect these polytopes at collections of three black points. If we assume that the lower left corner is $(0,0,0)$, these points have coordinates $\{(1,1,0),(1,0,1),(0,1,1)\}$, or
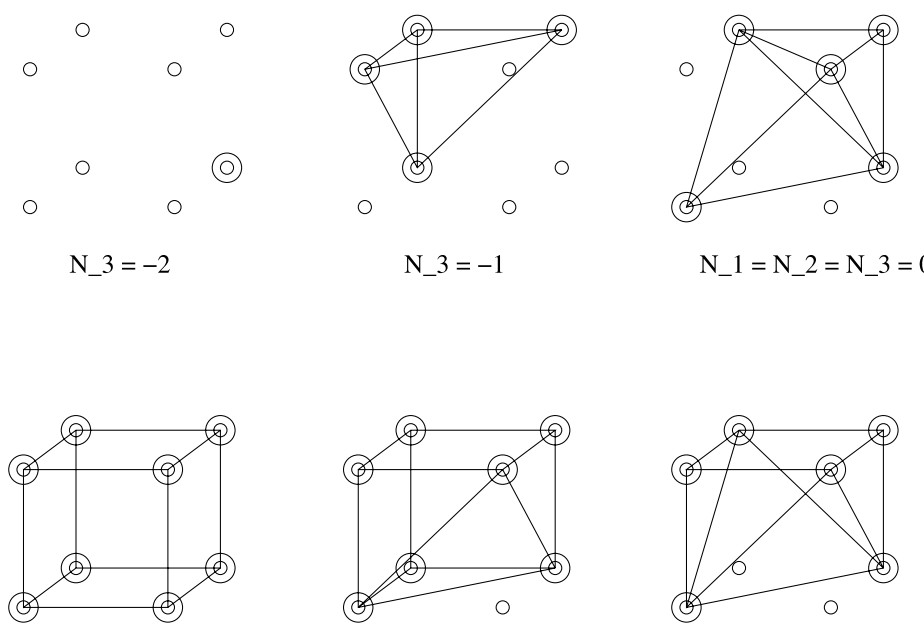

$$
\text { N_1, N_2, N_3 >0 }
$$

$\mathrm{N} \_1, \mathrm{~N} \_2>0, \mathrm{~N} \_3=0$

N_1 $>0, N \_2=N \_3=0$

Fig. 5 Primitive cube semigroups 
Fig. 6 Cube semigroups with the lattice $v_{1}+v_{2}+v_{3}=0 \bmod$ 2
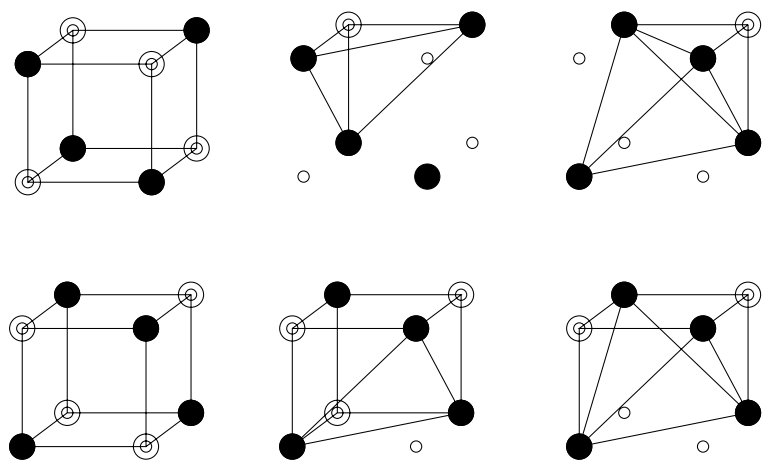

Fig. 7 New Possibilities for $C\left(m_{1}, m_{2}, m_{3}\right) \cap P_{3}(2 L)$
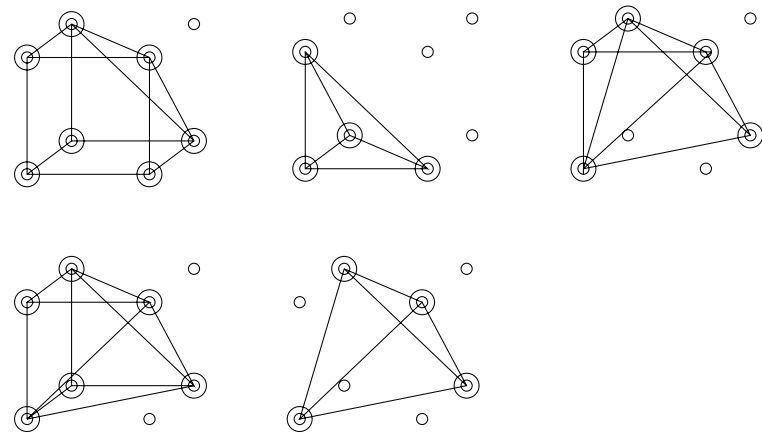

$\{(1,0,0),(0,1,0),(0,0,1)\}$. Figure 6 represents the new possibilities we must consider. The polytope pictured lower center in Figure 7 is the only case which is not lattice equivalent to one pictured in Figure 5 . It is rooted at $(0,0,0)$ and occurs only when $L=1$ (level condition is 2 ). The point $(1,1,1)$ in its second Minkowski sum cannot be expressed as the sum of two lattice points of degree one, so this is not a normal polytope. This is the reason we stipulate that $L>2$ in Theorem 1.9.

Now we analyze each $C\left(m_{1}, m_{2}, m_{3}\right) \cap P_{3}(2 L)$. Since lattice equivalent polytopes have isomorphic semigroups of lattice points, it suffices to investigate the polytopes listed in Figure 5.

Caution 2.1 In [3], Buczynska and Wisniewski study a normal polytope with the same vertices as the non-normal polytope mentioned above. This is possible because they are using the lattice $v_{1}+v_{2}+v_{3}=0 \bmod 2$, not the standard lattice.

\subsection{Graver Bases of the unit Cube}

We make use of the computational algebra package 4ti2, [1] to compute the Graver basis of the toric ideal of the unit 3-cube. Material on the Gröbner theory of toric 
ideals coming from polytopes can be found in [9].

$$
\begin{array}{ll}
(1,0,0)+(1,1,1)=(1,0,1)+(1,1,0) & (0,1,0)+(1,1,1)=(0,1,1)+(1,1,0) \\
(0,0,0)+(1,1,1)=(0,0,1)+(1,1,0) & (0,0,1)+(1,1,1)=(0,0,1)+(1,0,1) \\
(0,0,0)+(1,1,1)=(0,1,0)+(1,0,1) & (0,0,1)+(1,1,0)=(0,1,0)+(1,0,1) \\
(0,0,0)+(1,1,1)=(0,1,1)+(1,0,0) & (0,0,1)+(1,1,0)=(0,1,1)+(1,0,0) \\
(0,1,0)+(1,0,1)=(0,1,1)+(1,0,0) & (0,0,0)+(1,1,0)=(0,1,0)+(1,0,0) \\
(0,0,0)+(1,0,1)=(0,0,1)+(1,0,0) & (0,0,0)+(0,1,1)=(0,0,1)+(0,1,0)
\end{array}
$$

$$
\begin{aligned}
& (0,1,0)+(1,0,0)+(1,1,1)=(0,0,1)+(1,1,0)+(1,1,0) \\
& (0,0,0)+(1,1,1)+(1,1,1)=(0,1,1)+(1,0,1)+(1,1,0) \\
& (0,0,1)+(1,0,0)+(1,1,1)=(0,1,0)+(1,0,1)+(1,0,1) \\
& (0,0,1)+(0,0,1)+(1,1,0)=(0,0,0)+(0,1,1)+(1,0,1) \\
& (0,0,0)+(0,1,1)+(1,1,0)=(0,1,0)+(0,1,0)+(1,0,1) \\
& (0,0,0)+(1,0,1)+(1,0,1)=(0,1,1)+(1,0,0)+(1,0,0) \\
& (0,0,1)+(0,1,0)+(1,1,1)=(0,1,1)+(0,1,1)+(1,0,0) \\
& (0,0,0)+(0,0,0)+(1,1,1)=(1,0,0)+(0,1,0)+(0,0,1)
\end{aligned}
$$

Operating on this set of monomials, one can show that the toric ideal of every sub-polytope of the unit 3-cube which is not a simplex has a square-free Gröbner basis. This, combined with the fact that the sub-polytopes with $n_{3}=-2$ and -1 are unimodular simplices shows the following theorem.

Theorem 2.2 Suppose $L \neq 1$, then for all $\left(m_{1}, m_{2}, m_{3}\right)$, if $C\left(m_{1}, m_{2}, m_{3}\right) \cap P_{3}(2 L)$ is non-empty then it is a normal lattice polytope.

Remark 2.3 This theorem implies, among other things, that if $\omega \in U_{Y}^{2 L}[k]$, then

$$
\omega=\sum_{i=1}^{k} W_{i}
$$

for $W_{i} \in P_{3}(2 L)$ with the property that each

$$
W_{i}=X+\left(\epsilon_{1}, \epsilon_{2}, \epsilon_{3}\right)
$$

with $\epsilon_{j} \in\{0,1\}$ for all $i$ for a fixed $X \in \mathbb{R}^{3}$. It is easy to show that

$$
X=\left(\left\lfloor\frac{\omega(E)}{k}\right\rfloor,\left\lfloor\frac{\omega(F)}{k}\right\rfloor,\left\lfloor\frac{\omega(G)}{k}\right\rfloor\right) .
$$

Therefore each $W_{i}$ is $\left(\frac{\omega(E)}{k}, \frac{\omega(F)}{k}, \frac{\omega(G)}{k}\right)$ with either floor or ceiling applied to each entry.

Now we move on to relations. Let $S\left(m_{1}, m_{2}, m_{3}\right)$ be the semigroup of lattice points for $C\left(m_{1}, m_{2}, m_{3}\right) \cap P_{3}(2 L)-\left(m_{1}, m_{2}, m_{3}\right)$. Once again it suffices to treat the cases represented in Figure 5. 
Theorem 2.4 All relations for the semigroup $S\left(m_{1}, m_{2}, m_{3}\right)$ are reducible to quadrics and cubics.

Proof By Proposition 4.13 of [9], a Graver basis for any subpolytope $P$ of the unit 3cube is obtained by taking the members of the Graver basis of the unit 3-cube which have entries in the lattice points of $P$. Since these are all quadrics and cubics, we are done.

Up to equivalence and after accounting for redundancy, all relations considered here are of the form

$$
\begin{gathered}
(1,0,0)+(0,1,0)=(1,1,0)+(0,0,0) \\
(1,0,1)+(0,1,0)=(1,1,1)+(0,0,0) \\
(1,0,1)+(1,1,0)=(1,1,1)+(1,0,0) \\
(1,1,1)+(1,1,1)+(0,0,0)=(1,1,0)+(1,0,1)+(0,1,1),
\end{gathered}
$$

with the last one the only degree 3 relation, we refer to it as the "degenerated Segre Cubic" (see [6]).

\section{Proof of Theorem 1.9}

In this section we use Theorem 2.2 to prove that $U_{c(\mathcal{T})}^{L}(\mathbf{r})$ is generated in degree 1, which then proves Theorem 1.9. For each $v \in I(\mathcal{T})$ we have the morphism of graded semigroups

$$
i_{v}^{*}: U_{c(\mathcal{T})}^{L}(\mathbf{r}) \rightarrow U_{Y}^{L}
$$

Given a weight $\omega \in U_{c(\mathcal{T})}^{L}(\mathbf{r})$ we factor $i_{v}^{*}(\omega)$ for each $v \in I(c(\mathcal{T}))$ using Theorem 2.2. Then special properties of the weightings obtained by this procedure will allow us to glue the factors of the $i_{v}^{*}(\omega)$ back together along merged edges to obtain a factorization of $\omega$. First we must make sure that our factorization procedure does not disrupt the conditions at the edges of $c(\mathcal{T})$.

Lemma 3.1 Let $\omega \in U_{c(\mathcal{T})}^{L}(\mathbf{r})[k]$, and let $v \in I(\mathcal{T})$ be connected to a leaf of $c(\mathcal{T})$ at $E$. If $i_{v}^{*}(\omega)=\eta_{1}+\ldots+\eta_{k}$ is any factorization of $i_{v}^{*}(\omega)$ with $\eta_{i} \in$ $C\left(\left\lfloor\frac{i_{v}^{*}(\omega)(E)}{k}\right\rfloor,\left\lfloor\frac{i_{v}^{*}(\omega)(F)}{k}\right\rfloor,\left\lfloor\frac{i_{v}^{*}(\omega)(G)}{k}\right\rfloor\right)$, then $\eta_{i}(E)$ satisfies the appropriate edge condition for elements in $U_{c(\mathcal{T})}^{L}(\mathbf{r})[1]$.

Proof If $E$ is attached to a lone leaf of $\mathcal{T}$ then $i_{v}^{*}(\omega)(E)=k \mathbf{r}(e)$ for $i_{v}(E)=e$, $e \in V(\mathcal{T})$. By Remark 2.3

$$
\eta_{i}(E)=\lfloor\mathbf{r}(e)\rfloor=\mathbf{r}(e)
$$


or

$$
\eta_{i}(E)=\lfloor\mathbf{r}(e)\rfloor+1=\mathbf{r}(e)+1 .
$$

Since $\sum_{i=1}^{k} \eta_{i}(E)=k \mathbf{r}(e)$ we must have $\eta_{i}(E)=\mathbf{r}(e)$ for all $i$. If $E$ is a stalk of paired leaves $i$ and $j$ in $\mathcal{T}$ then we must have

$$
k \frac{|\mathbf{r}(i)-\mathbf{r}(j)|}{2} \leq \omega_{Y}(E) \leq k \frac{|\mathbf{r}(i)+\mathbf{r}(j)|}{2} .
$$

Note that both bounds are divisible by $k$. Since floor preserves lower bounds we have

$$
\frac{|\mathbf{r}(i)-\mathbf{r}(j)|}{2} \leq\left\lfloor\frac{i_{v}^{*}(\omega)(E)}{k}\right\rfloor,
$$

and since ceiling preserves upper bounds we have

$$
\left\lceil\frac{i_{v}^{*}(\omega)(E)}{k}\right\rceil \leq \frac{|\mathbf{r}(i)+\mathbf{r}(j)|}{2} .
$$

Therefore each $\eta_{i}$ satisfies

$$
\frac{|\mathbf{r}(i)-\mathbf{r}(j)|}{2} \leq \eta_{i}(E) \leq \frac{|\mathbf{r}(i)+\mathbf{r}(j)|}{2} .
$$

Now that we can safely use Theorem 2.2 with each $i_{v}^{*}: U_{c(\mathcal{T})}^{L}(\mathbf{r}) \rightarrow U_{Y}^{L}$, we will establish tools to extend factorization properties of $U_{Y}^{L}$ to $U_{c(\mathcal{T})}^{L}(\mathbf{r})$ by exploiting the fibered product structure of the ambient semigroup $U_{c(\mathcal{T})}^{L}$. The following concept allows us to control conditions on the edges of two trees we wish to merge.

Definition 3.2 We say that a list of nonnegative integers $\left\{X_{1}, \ldots, X_{n}\right\}$ is balanced if $\left|X_{i}-X_{j}\right|=1$ or 0 for all $i, j$.

Lemma 3.3 If two lists $\left\{X_{1}, \ldots, X_{n}\right\}$ and $\left\{Z_{1}, \ldots, Z_{m}\right\}$ are balanced, have the same total sum, and $n=m$, then they are the same list up to permutation.

Proof Let $C_{1}$ be the smallest member of $\left\{X_{1}, \ldots, X_{n}\right\}$, and $C_{2}$ be the smallest member of $\left\{Z_{1}, \ldots, Z_{n}\right\}$. Let $S$ be the total sum of either list. Both lists are balanced, so we must have $S=n C_{1}+k_{1}=n C_{2}+k_{2}$, where $k_{1}$ and $k_{2}$ are non-negative integers less than or equal to $n$. Suppose without loss of generality that $k_{2}-k_{1}$ is non-negative, then it must be divisible by $n$. By assumption, this can only happen if $k_{2}=k_{1}$, in which case $C_{1}=C_{2}$, and the lists have the same members.

Proposition 3.4 The semigroup $U_{c(\mathcal{T})}^{L}(\mathbf{r})$ is generated in degree 1 .

Proof Recall that by Remark 2.3, for any edge $E \in Y$ the edge weights of a factorization $i_{v}^{*}(\omega)=\eta_{1}+\ldots \eta_{k}$ satisfy $\eta_{i}(E)=\left\lfloor\frac{i_{v}^{*}(\omega)(E)}{k}\right\rfloor$ or $\left\lceil\frac{i_{v}^{*}(\omega)(E)}{k}\right\rceil$. Take any two $v_{1}$, $v_{2}$ which share a common edge $E$ in $c(\mathcal{T})$. Let $\omega \in U_{c(\mathcal{T})}^{L}(\mathbf{r})[k]$ and let $\left\{\eta_{1}^{1}, \ldots, \eta_{k}^{1}\right\}$ and $\left\{\eta_{1}^{2}, \ldots, \eta_{k}^{2}\right\}$ be factorizations of $i_{v_{1}}^{*}(\omega)$ and $i_{v_{2}}^{*}(\omega)$ respectively. Then the lists 
$\left\{\eta_{1}^{1}(E), \ldots, \eta_{k}^{1}(E)\right\}$ and $\left\{\eta_{1}^{2}(E), \ldots, \eta_{k}^{2}(E)\right\}$ are balanced and have the same sum, so by Lemma 3.3 they are the same list up to some permutation. We may glue factors $\eta_{i}^{1}$ and $\eta_{j}^{2}$ when $\eta_{i}^{1}(E)=\eta_{j}^{2}(E)$, the above observation guarantees that any $\eta_{i}^{1}$ has an available partner $\eta_{j}^{2}$. The proposition now follows by induction on the number of $v \in I(c(\mathcal{T}))$. This implies Theorem 1.9.

\section{Proof of Theorem 1.10}

In this section we show how to get all relations in $U_{c(\mathcal{T})}^{L}(\mathbf{r})$ from those lifted from $U_{Y}^{L}$. The procedure follows the same pattern as the proof of Theorem 1.9. We consider the image of a relation $\omega_{1}+\ldots+\omega_{n}=\eta_{1}+\ldots+\eta_{n}$ under a map $i_{v}^{*}: U_{c(\mathcal{T})}^{L}(\mathbf{r}) \rightarrow U_{Y}^{L}$, using Theorem 2.4 we convert this to a trivial relation using relations of degree at most 3. We then give a recipe for lifting each of these relations back to $U_{c(\mathcal{T})}^{L}(\mathbf{r})$. The result is a way to convert $\omega_{1}+\ldots+\omega_{n}=\eta_{1}+\ldots+\eta_{n}$ to a relation which is trivial over the trinode $v$ using quadrics and cubics. In this way we take a general relation to a trivial relation one $v \in I(c(\mathcal{T}))$ at a time.

Definition 4.1 A set of degree 1 elements $\left\{\omega_{1}, \ldots, \omega_{k}\right\}$ in $U_{c(\mathcal{T})}^{L}(\mathbf{r})$ is said to be balanced when the set $\left\{\omega_{1}(E), \ldots, \omega_{k}(E)\right\}$ is balanced for all $E \in c(\mathcal{T})$. A relation $\omega_{1}+\ldots+\omega_{k}=\eta_{1}+\ldots+\eta_{k}$ in $U_{c(\mathcal{T})}^{L}(\mathbf{r})$ is said to be balanced when $\left\{\omega_{1}, \ldots, \omega_{k}\right\}$ and $\left\{\eta_{1}, \ldots, \eta_{k}\right\}$ are balanced.

The following lemmas show that we need only consider balanced relations.

Lemma 4.2 Any list of non-negative integers $S=\left\{X_{1}, \ldots, X_{n}\right\}$ can be converted to a balanced list $T=\left\{Y_{1}, \ldots, Y_{n}\right\}$ with $\sum_{i=1}^{n} Y_{i}=\sum_{i=1}^{n} X_{i}$ by replacing a pair $X_{i}$ and $X_{j}$ with $\left\lfloor\frac{X_{i}+X_{j}}{2}\right\rfloor$ and $\left\lceil\frac{X_{i}+X_{j}}{2}\right\rceil$ a finite number of times.

Proof Let $d(S)$ be the difference between the maximum and minimum elements of $S$. It is clear that with a finite number of exchanges

$$
\left\{X_{i}, X_{j}\right\} \rightarrow\left\{\left\lfloor\frac{X_{i}+X_{j}}{2}\right\rfloor,\left\lceil\frac{X_{i}+X_{j}}{2}\right\rceil\right\}
$$

We get a new set $S^{\prime}$ with $d(S)>d\left(S^{\prime}\right)$, unless $d(S)=1$ or 0 . Since this happens if and only of $S$ is balanced, the lemma follows by induction.

\section{Lemma 4.3 Let}

$$
\omega_{1}+\ldots+\omega_{k}=\eta_{1}+\ldots+\eta_{k}
$$

be a relation in $U_{c(\mathcal{T})}^{L}(\mathbf{r})$. Then it can be converted to a balanced relation

$$
\omega_{1}^{\prime}+\ldots+\omega_{k}^{\prime}=\eta_{1}^{\prime}+\ldots+\eta_{k}^{\prime}
$$

using only degree 2 relations. 
Fig. 8 Component subtrees about a vertex

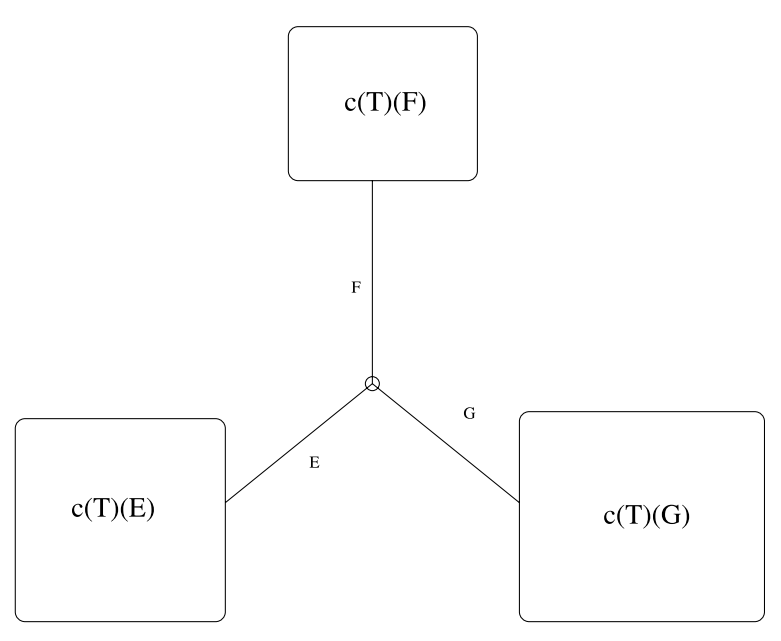

Proof First we note that we may use the proof of Theorem 1.9 to factor the weighting $\omega_{1}+\omega_{2}$ into $\omega_{1}^{\prime}+\omega_{2}^{\prime}$ so that $\left\{\omega_{1}^{\prime}, \omega_{2}^{\prime}\right\}$ is balanced. Using this and Lemma 4.2 we can find

$$
\omega_{1}^{\prime}+\ldots+\omega_{k}^{\prime}=\omega_{1}+\ldots+\omega_{k}
$$

such that the set $\left\{\omega_{1}^{\prime}(E), \ldots, \omega_{k}^{\prime}(E)\right\}$ is balanced for some specific $E$, employing only degree 2 relations. Observe that if $\left\{\omega_{1}(F), \ldots, \omega_{k}(F)\right\}$ is balanced for some $F$, the same is true for $\left\{\omega_{1}^{\prime}(F), \ldots, \omega_{k}^{\prime}(F)\right\}$, after a series of degree 2 applications of 1.9. This shows that we may inductively convert $\left\{\omega_{1}, \ldots, \omega_{k}\right\}$ to $\left\{\omega_{1}^{\prime}, \ldots, \omega_{k}^{\prime}\right\}$ with the property that $\left\{\omega_{1}^{\prime}(E), \ldots, \omega_{k}^{\prime}(E)\right\}$ is a balanced list for all edges $E$, using only degree 2 relations. Applying the same procedure to the $\eta_{i}$ then proves the lemma.

The next lemma shows how we lift a balanced relation in $U_{Y}^{L}$ to one in $U_{c(\mathcal{T})}^{L}(\mathbf{r})$.

Lemma 4.4 Let $\left\{\omega_{1} \ldots \omega_{k}\right\}$ be a balanced set of elements in $U_{c(\mathcal{T})}^{L}(\mathbf{r})$. Let $i_{v}^{*}\left(\omega_{1}\right)+$ $\ldots+i_{v}^{*}\left(\omega_{k}\right)=\eta_{1}+\ldots+\eta_{k}$ be a balanced degree $k$ relation in the appropriate $S\left(m_{1}, m_{2}, m_{3}\right) \subset U_{Y}^{L}$. Then the $\eta_{i}$ may be lifted to weightings of $c(\mathcal{T})$ giving a relation of degree $k$ in $U_{c(\mathcal{T})}^{L}(\mathbf{r})$ which agrees with the relation above when $i_{v}^{*}$ is applied, and is a permutation of $i_{v^{\prime}}^{*}\left(\omega_{1}\right) \ldots i_{v^{\prime}}^{*}\left(\omega_{k}\right)$ for $v^{\prime} \neq v$.

Proof Let $c(\mathcal{T})(E)$ be the unique connected subtrivalent tree of $c(\mathcal{T})$ which includes $v$ and has the property that any path $\gamma \subset c(\mathcal{T})(E)$ with endpoints at a vertex $v^{\prime} \neq v$ in $c(\mathcal{T})(E)$ and $v$ includes the edge $E$ (see Figure 8), define $c(\mathcal{T})(F)$ and $c(\mathcal{T})(G)$ in the same way. To make $\eta_{1}^{\prime} \ldots \eta_{k}^{\prime}$ over $c(\mathcal{T})$, note that the list $\left\{i_{c(\mathcal{T})(E)}^{*}\left(\omega_{i}\right)(E)\right\}$ is the same as the list $\left\{\eta_{i}(E)\right\}$ up to permutation, because they are both balanced lists with the same sum and the same number of elements, so we may glue these weightings together to make a tuple over $c(\mathcal{T})$. 
Suppose we are given a balanced relation

$$
\omega_{1}+\ldots+\omega_{k}=\eta_{1}+\ldots+\eta_{k} .
$$

We can pick any $v \in I(c(\mathcal{T}))$, and consider the relation

$$
i_{v}^{*}\left(\omega_{1}\right)+\ldots+i_{v}^{*}\left(\omega_{k}\right)=i_{v}^{*}\left(\eta_{1}\right)+\ldots+i_{v}^{*}\left(\eta_{k}\right)
$$

We convert this to a trivial relation using a series of relations in the appropriate $S\left(m_{1}, m_{2}, m_{3}\right)$, then lift the result back to $U_{c(\mathcal{T})}^{L}(\mathbf{r})$. For any $v^{\prime} \neq v$ in $I(c(\mathcal{T}))$, this process only permutes the members of $\left\{i_{v^{\prime}}^{*}\left(\omega_{1}\right), \ldots, i_{v^{\prime}}^{*}\left(\omega_{k}\right)\right\}$ and $\left\{i_{v^{\prime}}^{*}\left(\eta_{1}\right), \ldots, i_{v^{\prime}}^{*}\left(\eta_{k}\right)\right\}$, which does not change whether or not this was a balanced relation. In this way, we may convert any balanced relation in $U_{c(\mathcal{T})}^{L}(\mathbf{r})$ to a trivial relation one $v \in I(c(\mathcal{T}))$ at a time.

Proposition 4.5 Let $N$ be the maximum degree of relations needed to generate all relations in the semigroups $S\left(m_{1}, m_{2}, m_{3}\right)$. Then the semigroup $U_{c(\mathcal{T})}^{L}(\mathbf{r})$ has relations generated in degree bounded by $N$.

This proposition, coupled with Theorem 2.4 proves Theorem 1.10 . We recap the content of the last two sections with the following theorem.

Theorem 4.6 Let $(\mathcal{T}, \mathbf{r}, L)$ be admissible. Then the ring $\mathbb{C}\left[U_{c(\mathcal{T})}^{L}(\mathbf{r})\right]$ has a presentation

$$
0 \longrightarrow I \longrightarrow \mathbb{C}[X] \longrightarrow \mathbb{C}\left[U_{c(\mathcal{T})}^{L}(\mathbf{r})\right] \longrightarrow 0
$$

where $X$ is the set of degree 1 elements of $U_{c(\mathcal{T})}^{L}(\mathbf{r})$, and I is the ideal generated by two types of binomials,

$$
\left[\omega_{1}\right] \circ \ldots \circ\left[\omega_{n}\right]-\left[\eta_{1}\right] \circ \ldots \circ\left[\eta_{n}\right]
$$

(1) Binomials where $n \leq 3, i_{v}^{*}\left(\omega_{1}\right)+\ldots+i_{v}^{*}\left(\omega_{n}\right)=i_{v}^{*}\left(\eta_{1}\right)+\ldots+i_{v}^{*}\left(\eta_{n}\right)$ is a balanced relation in $U_{Y}^{L}$ for some specific $v$, and $\left\{i_{v^{\prime}}^{*}\left(\omega_{1}\right), \ldots, i_{v^{\prime}}^{*}\left(\omega_{n}\right)\right\}=$ $\left\{i_{v^{\prime}}^{*}\left(\eta_{1}\right), \ldots, i_{v^{\prime}}^{*}\left(\eta_{n}\right)\right\}$ for $v \neq v^{\prime}$.

(2) Binomials where $n=2$ and $i_{v}^{*}\left(\omega_{1}\right)+i_{v}^{*}\left(\omega_{2}\right)=i_{v}^{*}\left(\eta_{1}\right)+i_{v}^{*}\left(\eta_{2}\right)$ such that $\left\{i_{v}^{*}\left(\omega_{1}\right), i_{v}^{*}\left(\omega_{2}\right)\right\}$ is balanced for all $v \in I(c(\mathcal{T}))$.

This induces a presentation for $\mathbb{C}\left[S_{\mathcal{T}}^{L}(\mathbf{r})\right]$ by isomorphism.

Corollary 4.7 The same holds for $\mathbb{C}\left[S_{\mathcal{T}}(\mathbf{r})\right]$.

Proof For each pair $(\mathcal{T}, \mathbf{r})$ it is easy to show that there is a number $N(\mathcal{T}, \mathbf{r})$, such that any weighting $\omega$ which satisfies the triangle inequalities on $\mathcal{T}$ and has $\omega\left(e_{i}\right)=$ $\mathbf{r}_{i}$ must have $\omega(e) \leq N(\mathcal{T}, \mathbf{r})$ for $e \in E(\mathcal{T})$. Because of this $S_{\mathcal{T}}^{L}(\mathbf{r})=S_{\mathcal{T}}(\mathbf{r})$ for $L$ sufficiently large. 


\section{Special Cases and Observations}

In this section we collect results on some special cases of $\mathbb{C}\left[S_{\mathcal{T}}^{L}(\mathbf{r})\right]$. In particular we study some instances when cubic relations are unnecessary, we give some examples where the semigroup is not generated in degree 1, we analyze the case when $L$ is allowed to be odd, and we give instances where cubic relations are necessary.

\subsection{The Caterpillar Tree}

One consequence of the proof of Theorem 2.4 is that a semigroup $U_{c(\mathcal{T})}^{L}(\mathbf{r})$ which omits or only partially admits the semigroup $S(0,0,0)$ or $S(L-1, L-1,0)$ as an image of one of the morphisms $i_{v}^{*}$ manages to avoid degree 3 relations entirely. The next proposition illustrates one such example, the semigroups of weightings on the caterpillar tree, pictured below.

Proposition 5.1 Let $\mathcal{T}_{0}$ be the caterpillar tree, and let $\mathbf{r}(i)$ be even for all $i \in V\left(\mathcal{T}_{0}\right)$. Then $S_{\mathcal{T}_{0}}^{2 L}(\mathbf{r})$ is generated in degree 1 , with relations generated by quadrics.

Proof We catalogue the weights $i_{v}^{*}(\omega)$ which can appear in degree 1 . For the sake of simplicity we divide all weights by 2 . Suppose $i_{v}(G)$ is an external edge, then $i_{v}^{*}(\omega)(E)$ and $i_{v}^{*}(\omega)(F)$ satisfy the following inequalities

$$
\begin{gathered}
i_{v}^{*}(\omega)(E) \leq i_{v}^{*}(\omega)(F)+\mathbf{r}(i) \\
i_{v}^{*}(\omega)(F) \leq i_{v}^{*}(\omega)(E)+\mathbf{r}(i) \\
i_{v}^{*}(\omega)(E)+i_{v}^{*}(\omega)(F)+\mathbf{r}(i) \leq 2 L
\end{gathered}
$$

where $i_{v}^{*}(\omega)(G)=\mathbf{r}(i)$. These conditions define a polytope in $\mathbb{R}^{2}$ with vertices $(L, L-\mathbf{r}(i)),(L-\mathbf{r}(i), L),(\mathbf{r}(i), 0)$ and $(0, \mathbf{r}(i))$. Pictured below is the case $L=9$, $2 \mathbf{r}(i)=6$.

When two edges are external, the polytope is an integral line segment. Note that the intersection of any lattice cube in $\mathbb{R}^{2}$ with the above polytope is a simplex or a unit square. Both of these polytopes have at most quadrics for relations in their semigroup of lattice points. Hence the argument used to prove Theorem 1.10 shows that $U_{c\left(\mathcal{T}_{0}\right)}^{2 L}(\mathbf{r})$ needs only quadric relations.

Fig. 9 The Caterpillar tree

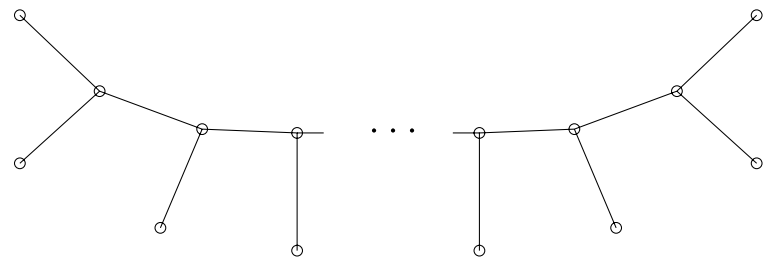


Fig. 10 The case $L=9$, $2 \mathbf{r}(i)=6$

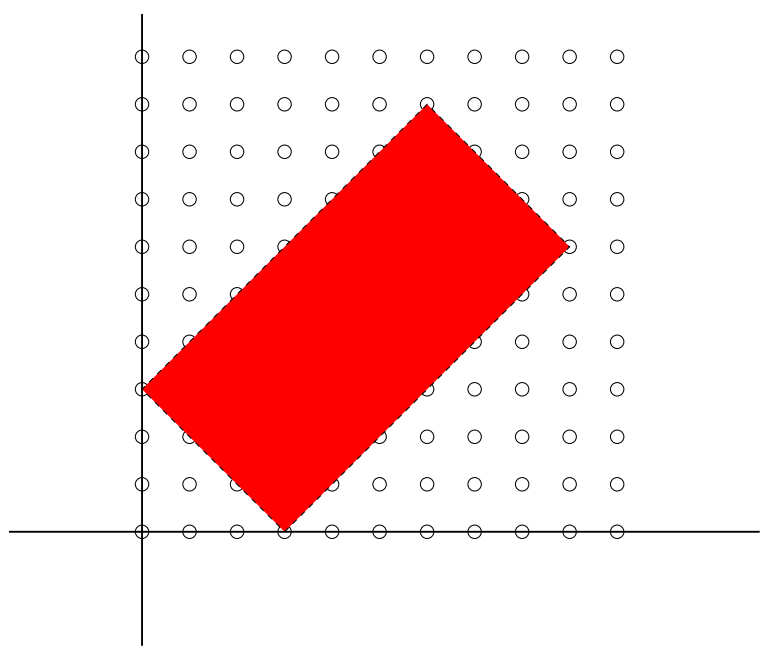

Corollary 5.2 If $L>1$, and $\mathbf{r}$ is a vector of nonnegative integers, the ring $R^{G}(2 L, 2 \mathbf{r})$ has a presentation with defining ideal generated by quadrics. In particular, the second Veronese subring of any $R^{G}(\mathbf{r}, L)$ has such a presentation if $L>1$. Note that the same applies to $\mathbb{C}\left[\mathcal{M}_{2 \mathbf{r}}\right]$.

\subsection{Counterexamples to Degree 1 generation}

Now we'll see examples of $(\mathbf{r}, \mathcal{T}, L)$ such that $S_{\mathcal{T}}^{L}(\mathbf{r})$ is not generated in degree 1 . We will begin by defining a certain class of paths in the tree $\mathcal{T}$. Let $\mathcal{T}$ have an even number of leaves. We claim that there is a set of edges $A(\mathcal{T}) \subset E(\mathcal{T})$ the members of which are assigned odd numbers by any weighting $\omega$ which assigns an odd number to each leaf of $\mathcal{T}$. It suffices to establish the stronger result that the parity of members of $V(\mathcal{T})$ determines the parity of every edge in $\mathcal{T}$. To see this, first note that the parity of two edges of a trinode determines the parity of the third edge, an induction argument on the number of edges in $\mathcal{T}$ does the rest.

Proposition 5.3 Let $\mathcal{T}$ be as above. The set $A(\mathcal{T})$ is a union of edges from disjoint paths in $\mathcal{T}$.

Proof Exactly two out of three edges in each trinode can be assigned an odd number, by the parity condition. This establishes the proposition.

From now on we let $O(\mathcal{T})$ denote the set of paths established by the previous proposition.

Proposition 5.4 Let $(\mathbf{r}, \mathcal{T}, L)$ be such that the edges connected to the endpoints of each member of $O(\mathcal{T})$ are given the same parity by $\mathbf{r}$. Assume further that there is a $\gamma \in O(\mathcal{T})$ such that end points of $\gamma$ are connected to edges $e$ and $f$ with $\mathbf{r}(e)$ and $\mathbf{r}(f)$ odd. If there is a degree 2 weighting which assigns 0 to any edge in $\gamma$, then $S_{\mathcal{T}}^{2 L}(\mathbf{r})$ is not generated in degree 1. 
Fig. 11 E2 and E3 are lone leaves connected by an element of $O(\mathcal{T})$

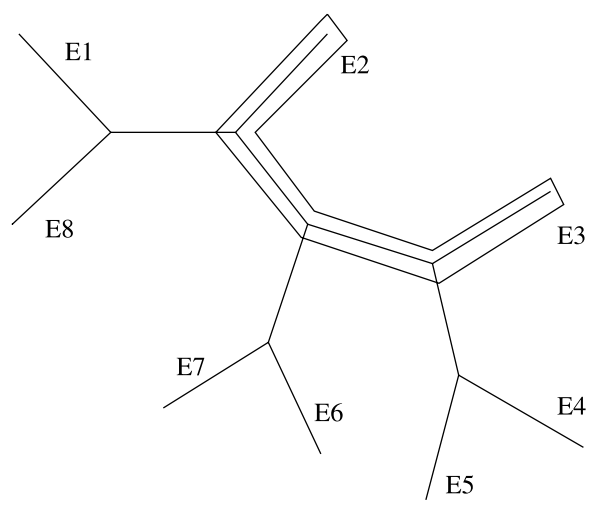

Proof All degree 1 elements must assign odd numbers to the edges in $\gamma$. No two odd numbers add to 0 .

Corollary 5.5 For simplicity, let $L>1$. Let $\overrightarrow{1}$ be the $n$-vector of 1 's. The semigroup $S_{\mathcal{T}}^{2 L}(\overrightarrow{1})$ is generated in degree 1 if and only if $\mathcal{T}$ has the property that no leaf is lone.

Proof First note that the if portion of this statement is taken care of by Theorem 1.9. Suppose now that $\mathcal{T}$ has lone leaves. Then two of these leaves are connected by a member $\gamma$ of $O(\mathcal{T})$. Pick any non-leaf edge $e$ in $\gamma$, and consider the weighting $\omega$ which assigns 0 to $e$ and 2 to every other edge in $\mathcal{T}$. We have $\omega \in S_{\mathcal{T}}^{2 L}(\overrightarrow{1})$ [2] for any $L$, and by Proposition $5.4 \omega$ cannot be factored.

Remark 5.6 Trees with the property that no leaf is lone are called good trees in [6], where they were introduced for the purpose of proving the analogue of Corollary 5.5 for $S_{\mathcal{T}}(\overrightarrow{1})$.

\subsection{The Case when $L$ is odd}

When the level $L$ is odd, the polytope $P_{3}(L)$ is no longer integral, however its Minkowski square $P_{3}(2 L)$ is integral, so clearly there are elements of $P_{3}(2 L)$ which cannot be integrally factored, specifically the corners. This observation has a generalization.

Definition 5.7 Let I $P_{3}(L)$ be the convex hull of the integral points of $P_{3}(L)$. Let $\Omega$ be the set of elements in the graded semigroup of lattice points of $P_{3}(L)$ such that $\frac{1}{\operatorname{deg}(Q)} Q \in P_{3}(L) \backslash I P_{3}(L)$.

Let $(E, F, G)=Q \in P_{3}(L)$ be integral with $L$ odd, and suppose $E, F$, or $G \geq$ $\frac{L-1}{2}+1$. Then, by the triangle inequalities we must have $F+G \geq \frac{L-1}{2}+1$, so $E+F+G \geq L+1$, a contradiction. This shows that $I P_{3}(L)$ is contained in the intersection of $P_{3}(L)$ with the halfspaces defined by $E, F, G \leq \frac{L-1}{2}$, this identifies 
Fig. 12 The Polytope $I P_{3}(5)$

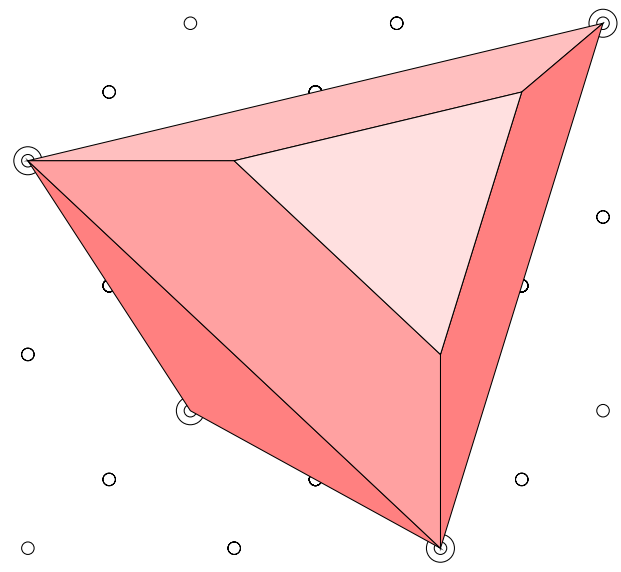

I $P_{3}(L)$ as the convex hull of the set

$$
\begin{gathered}
\left\{(0,0,0),\left(\frac{L-1}{2}, \frac{L-1}{2}, 0\right),\left(\frac{L-1}{2}, 0, \frac{L-1}{2}\right),\left(0, \frac{L-1}{2}, \frac{L-1}{2}\right),\right. \\
\left.\left(\frac{L-1}{2}, \frac{L-1}{2}, 1\right),\left(\frac{L-1}{2}, 1, \frac{L-1}{2}\right),\left(1, \frac{L-1}{2}, \frac{L-1}{2}\right)\right\} .
\end{gathered}
$$

The polytope $I P_{3}(5)$ is pictured above.

Proposition 5.8 Any $Q \in \Omega$ cannot be integrally factored.

Proof This follows from the observation that if $Q=E_{1}+\ldots+E_{n}$ then $\frac{1}{n} Q$ is in the convex hull of $\left\{E_{1}, \ldots, E_{n}\right\}$.

A factorization of any element $\omega$ such that $i_{v}^{*}(\omega)=Q$ gives a factorization of $Q$. So any $\omega \in U_{c(\mathcal{T})}^{L}(\mathbf{r})$ with a $i_{v}^{*}(\omega) \in \Omega$ is necessarily an obstruction to generation in degree 1 , this also turns out to be a sufficient obstruction criteria.

Theorem 5.9 Let $\mathcal{T}$ and $\mathbf{r}$ satisfy the same conditions as admissibility, and let $L \neq 2$. Then $U_{c(\mathcal{T})}^{L}(\mathbf{r})$ is generated in degree 1 if and only if

$$
i_{v}^{*}(\omega) \in U_{Y}^{L} \backslash \Omega
$$

for all $v \in I(c(\mathcal{T})), \omega \in U_{c(\mathcal{T})}^{L}(\mathbf{r})$. In this case all relations are generated by those of degree at most 3 .

Proof We analyze $I P_{3}(L)$ in the same way we did $P_{3}(2 L)$. The reader can verify that the integral points of $C\left(m_{1}, m_{2}, m_{3}\right) \cap P_{3}(L)$ are the same as the integral points of $C\left(m_{1}, m_{2}, m_{3}\right) \cap I P_{3}(L)$. The possibilities are represented by slicing the cubes in Figure 6 along the plane formed by the upper right or lower left collection of three non-filled dots, depending on the cube, and then restricting to the convex hull of the 
remaining integral points. All cases are lattice equivalent to one of the polytopes listed in Figure 5, after considering two- and one-dimensional cases as faces of neighboring three-dimensional polytopes. Since any element of $U_{Y}^{L}$ not in $\Omega$ is necessarily a lattice point of a Minkowski sum of $I P_{3}(L)$, the theorem follows by the same arguments used to prove Theorems 1.9 and 1.10 .

\subsection{Necessity of Degree 3 Relations}

Now we show that there are large classes of admissible $(\mathcal{T}, \mathbf{r}, L)$ which require degree 3 relations. We will exhibit a degree 3 weighting which has only two factorizations. The tree $\mathcal{T}$ with weight $\omega_{\mathcal{T}}$ is pictured below, it is an element of $S_{\mathcal{T}}(\overrightarrow{2})$. In all that follows all weightings are considered to have been halved.

Notice that $\omega_{\mathcal{T}}$ has 3 -way symmetry about the central trinode, we will exploit this by considering the tree $\mathcal{T}^{\prime}$ with restricted weighting $\omega_{\mathcal{T}}$, pictured in Figure 14 . We find the weightings that serve as a degree 1 factors of $\omega_{\mathcal{T}}$. First of all, any degree 1 weighting which divides $\omega_{\mathcal{T}}$, must be as in Figure 15 .

It suffices to find the possible values of $X$ and $Z$. Both must be less than or equal to 2 , which shows that $Z$ can be either 2 or 1 . This implies that two factors have $Z=2$

Fig. $13 \omega_{\mathcal{T}}$

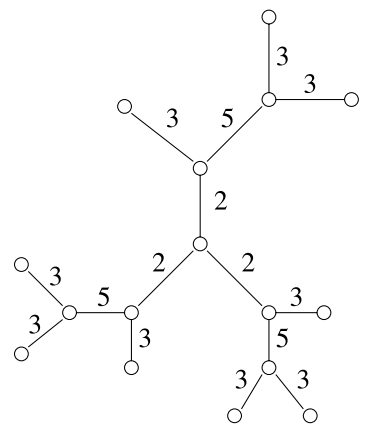

Fig. $14 \omega_{\mathcal{T}^{\prime}}$

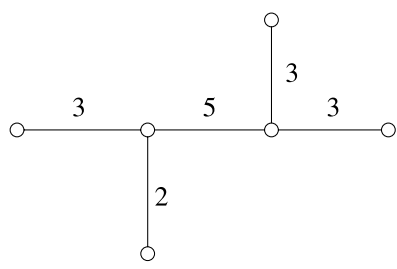

Fig. 15

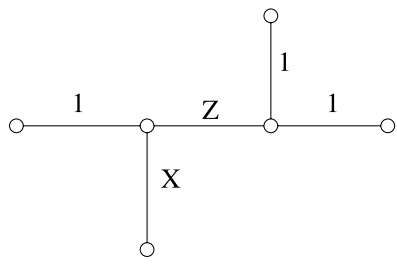


Fig. 16
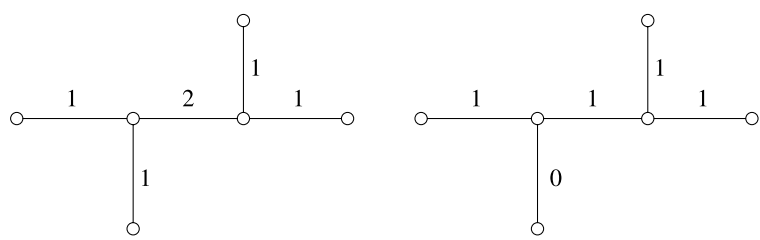

Fig. 17 Grafting two tree weightings
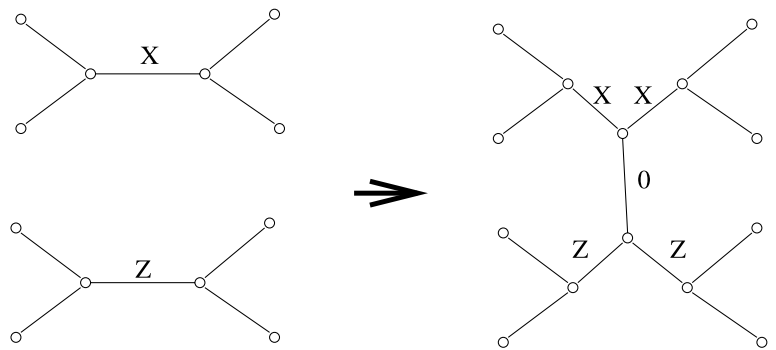

and one factor has $Z=1$. For $X$, we note that $X=0$ cannot be paired with $Z=2$ because of the triangle inequalities. This implies that $X$ cannot equal 2 , and that both factors with $Z=2$ have $X=1$, and $Z=1$ is paired with $X=0$. This shows that there are exactly two possibilities determined by the value of $X$, both are shown in Figure 16. Any factorization of $\omega_{\mathcal{T}}$ is determined by its values on the central trinode, and these values must be weights composed entirely of 0 and 1 . There are exactly two such variations, making the Degenerated Segre Cubic.

We have not specified a level $L$ for this weighting, but the same argument applies for any level large enough to admit $\omega_{\mathcal{T}}$ as a weighting in degree 3 . For any tree $\mathcal{T}^{*}$, edge $e^{*} \in$ tree $^{*}$, and weight $\omega_{\mathcal{T}} *$ we can create a new weight on a larger tree by adding a vertex in the middle of $e^{*}$, attaching a new leaf edge at that vertex, and weighting the both sides of the split $e^{*}$ with $\omega_{\mathcal{T}^{*}}\left(e^{*}\right)$, and the new edge with 0 . Using this procedure on any $\left(\mathcal{T}^{*}, e^{*}, \omega_{\mathcal{T}} *\right)$, and $\left(\mathcal{T}, e, \omega_{\mathcal{T}}\right)$ for any edge $e \in \mathcal{T}$, can create a new weighted tree by identifying the new 0 -weighted edges. On the level of the combinatorics of the trees, this construction is called the graft of two pointed trees, and was introduced in Definition 2.25 of [3]. An example is pictured in Figure 17. In this way many examples of unremovable degree 3 relations can be manufactured.

Open Access This article is distributed under the terms of the Creative Commons Attribution Noncommercial License which permits any noncommercial use, distribution, and reproduction in any medium, provided the original author(s) and source are credited.

\section{References}

1. 4ti2 Team: 4ti2, A software package for algebraic, geometric and combinatorial problems on linear spaces. Available at www.4ti2.de

2. Bauer, S.: Parabolic bundles, elliptic surfaces and SU(2)-representation spaces of genus zero Fuchsian groups. Math. Ann. 290, 509-526 (1991)

3. Buczynska, W., Wisniewski, J.: On the geometry of binary symmetric models of phylogenetic trees. J. Eur. Math. Soc. 9, 609-635 (2007) 
4. Castravet, A.-M., Tevelev, J.: Hilbert's 14th problem and Cox rings. Compos. Math. 142, 1479-1498 (2006)

5. Howard, B.J., Manon, C.A., Millson, J.J.: The toric geometry of triangulated polygons in Euclidean space. Can. J. Math., to appear.

6. Howard, B.J., Millson, J.J., Snowden, A., Vakil, R.: The projective invariants of ordered points on the line. arXiv:math.AG/0505096

7. Mukai, S.: Geometric realization of $T$-shaped root systems and counterexamples to Hilbert's fourteenth problem. In: Algebraic Transformation Groups and Algebraic Varieties. Encyclopaedia Math. Sci., vol. 132, pp. 123-129. Springer, Berlin (2004). MR2090672

8. Speyer, D., Sturmfels, B.: The tropical Grassmannian. Adv. Geom. 4, 389-411 (2004) arXiv: math.AG/0304218

9. Sturmfels, B.: Gröbner Bases and Convex Polytopes. University Lecture Series, vol. 8, American Mathematical Society, Providence (1996)

10. Sturmfels, B., Xu, Z.: Sagbi bases of Cox-Nagata rings. J. Eur. Math. Soc., to appear. arXiv:0803.0892v2 [math.AG] 